\title{
Multidisciplinary Team Care in the Surgical Management of Pituitary Adenoma
}

\author{
Jessica W. Grayson ${ }^{1}$ Agnish Nayak ${ }^{1}$ Mark Winder ${ }^{2}$ Benjamin Jonker ${ }^{2,3,4}$ Raquel Alvarado ${ }^{1}$ \\ Henry Barham ${ }^{5}$ Ann McCormack $6,7,8$ Richard J. Harvey ${ }^{1,9}$
}

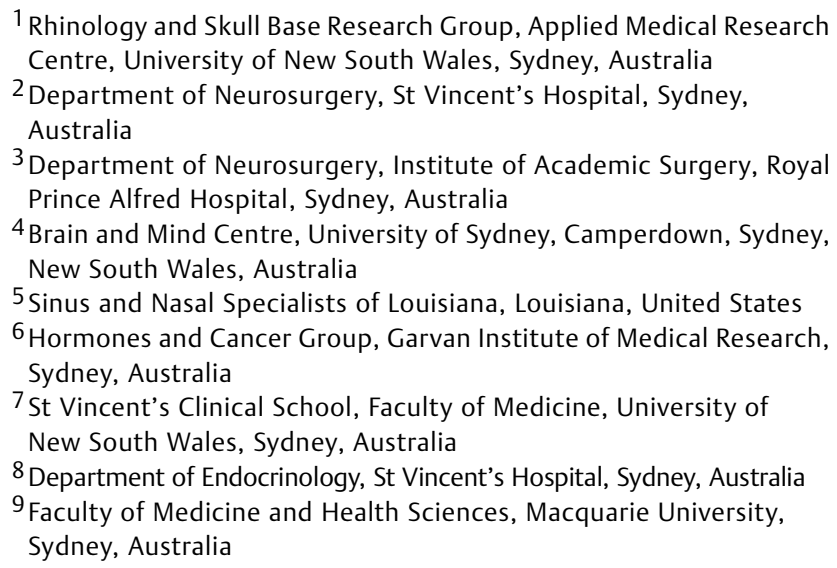

Address for correspondence Jessica Grayson, MD, 67 Burton Street, Darlinghurst 2010, New South Wales, Australia (e-mail: jessica.w.grayson@gmail.com).

J Neurol Surg B 2021;82:295-302.

\begin{abstract}
\section{Keywords}

- multidisciplinary care

- pituitary surgery

- syndrome of inappropriate antidiuretic hormone

- diabetes insipidus

- length of stay

Objective Despite multidisciplinary care being commonly recommended, there remains limited evidence supporting its benefits in pituitary disease management. This study aimed to assess the impact of multidisciplinary care in pituitary surgery.

Methods A retrospective cohort study was performed comparing pituitary surgery outcomes among consecutive patients within a quaternary referral center in 5 years before and after introduction of a multidisciplinary team (MDT). Primary outcomes were endocrine (transient diabetes insipidus [DI], syndrome of inappropriate antidiuretic hormone $[\mathrm{SIADH}]$, and new hypopituitarism) and surgical (cerebrospinal fluid [CSF] leak, epistaxis, intracranial hemorrhage, and meningitis) complications, length of hospital stay, and intrasellar residual tumor.

Results 279 patients ( 89 pre-MDT vs. 190 post-MDT) were assessed (age $54 \pm 17$ years, $48 \%$ female). Nonfunctioning adenomas were most common (54\%). In the post-MDT era, more clinically functioning tumors ( 42 vs. $28 \%, p=0.03$ ) were treated. Transient $\mathrm{DI}$ and SIADH occurred less often post-MDT ( 20 vs. $36 \%, p<0.01$ and 18 vs. $39 \%, p<0.01$ ), as well as new hypothyroidism ( 5 vs. $15, p<0.01$ ). Hospital stay was shorter post-MDT (5[3] vs. 7 [5] days, $p<0.001$ ) and intrasellar residuals were less common (8vs. 35\%, $p<0.001$ ). Complications were more frequent pre-MDT independent of tumor size, hormone status, and surgical technique (odds ratio $[O R]=2.14$ [1.05-4.32], $p=0.04$ ).

Conclusion Outcomes of pituitary surgery improved after the introduction of an MDT. Pituitary MDTs may benefit both patients and the health system by improving quality of care and reducing hospital stays.
\end{abstract}

received

June 15, 2019

accepted after revision

September 13, 2019

published online

October 21, 2019 (c) 2019. Thieme. All rights reserved.

Georg Thieme Verlag KG,

Rüdigerstraße 14,

70469 Stuttgart, Germany
DOI https://doi.org/ 10.1055/s-0039-1700498. ISSN 2193-6331. 


\section{Introduction}

Multidisciplinary teams (MDTs) are frequently utilized in the management of a wide variety of cancers including breast, colorectal, lung, gynecological, and upper gastrointestinal malignancies. ${ }^{1}$ In the past years, specialists primarily liaised with each other on a consultation basis upon request. The newer concept of a regular, formal MDT meeting offers the opportunity for improved communication, development of cohesive management plans, and an educational platform between treating clinicians. However, this model of care, often recommended in clinical practice guidelines, incurs significant costs in terms of time and resources. ${ }^{2,3}$ The cost to the health system of a 2-hour monthly pituitary MDT meeting has been estimated to be $£ 9,000$ to 12,000 per year in the United Kingdom. ${ }^{4}$ While commonly used, the efficacy and cost-effectiveness of MDT models has not been well described. ${ }^{1,5}$

The mode of operation of MDT teams varies internationally. However, generally these follow a principle of initial case referral to the team or one of its members, subsequent discussion of cases at a regular interval (e.g., weekly or fortnightly), initiation of treatment through most relevant specialties and review by the team at a set date. ${ }^{3,6}$ Pituitary MDTs have only been implemented in recent years within some large pituitary quaternary referral centers.

Organizing multidisciplinary clinical management is a key criterion in defining pituitary tumor centers of excellence as per the recently published Pituitary Society guidelines. ${ }^{7}$ It is suggested that these teams require dedicated endocrinologists and experienced neurosurgeons working in collaboration with neuroradiology, neuropathology, radiation oncology, neuroophthalmology, and otorhinolaryngology plus trained nursing.

Despite pituitary MDTs becoming more common, the literature suggests there is little comparative evidence supporting their establishment. Pituitary MDTs are often briefly mentioned as being beneficial for outcomes in pituitary surgery by anecdotal evidence. ${ }^{8-26}$ This is partly attributed to better engagement of relevant specialties to facilitate diagnosis, prevent complications, and utilize the latest developments in practice. However, substantive evidence is limited, with few studies comparing complication and success rates before and after the introduction of MDT management. ${ }^{27}$

A recent retrospective cohort study found that the introduction of pituitary MDT care reduced length of hospital stay postoperatively from a median of 3 to 2 days that was attributed to endocrinologist involvement on a regular basis as part of an early discharge protocol. In this study, complication rates remained equivalent. ${ }^{27}$ It should be noted, however, that this team only comprised of a neurosurgeon, endocrinologist, endocrinology fellow, and specialty nurse. Thus, additional evidence is necessary to support the benefit of collaboration between broader group of disciplines given the increased associated cost of a larger MDT.

The objective of this study was to determine the impact of multidisciplinary care review in pituitary patients by outcome-based comparison before and after the introduction of an MDT.

\section{Methods}

A retrospective cohort study was performed comparing consecutive patients who underwent pituitary surgery before and after the introduction of a pituitary MDT, servicing both St. Vincent's Hospital and St Vincent's Private Hospital, Sydney. The pituitary MDT was implemented in 2012 and consists of members from endocrinology $(n=2)$, neurosurgery $(n=2)$, otolaryngology $(n=1)$, radiation and medical oncology ( $n=1$ each), radiology $(n=1)$, pathology $(n=1)$, ophthalmology $(n=1)$, nursing $(n=1)$, fellows, medical students, and pituitary research staffs. One important initiative established by the pituitary MDT was a perioperative protocol to guide postoperative steroid and diabetes insipidus (DI) management.

Pituitary surgical patients were divided into two groups, (1) those undergoing surgery prior to the introduction of the MDT (2006-2011; "pre-MDT"), and (2) those undergoing surgery after the introduction of the MDT (2012-2017; "post-MDT"). This study was approved by the local St. Vincent's Hospital Human Research Ethics Committee (SVH 13/033 and SVH 14/060) and has been performed in accordance with the ethical standards as laid down in the 1964 Declaration of Helsinki and its later amendments or comparable ethical standards.

\section{Management of MDT Patients}

Pituitary MDT meetings are held fortnightly. Patients are referred to the pituitary MDT via lead endocrinologist and MDT chair (A.M). Case mix includes patients with diagnostic challenges, where surgery or radiation therapy is being considered, all surgical cases within the first 2 weeks and at 3 months postoperatively, as well as patients with aggressive pituitary tumors, or carcinomas receiving oncological treatment. Review of all relevant clinical information, imaging, ophthalmic assessments, and histopathology is undertaken at the MDT with medical and/or surgical treatment options discussed and recorded. Patients recommended to undergo surgical intervention are then evaluated by neurosurgery, otolaryngology, and ophthalmology (where appropriate). During their hospitalization postoperatively, all patients were evaluated regularly by our endocrinology team with reference to our perioperative pituitary protocol.

\section{Medical Follow-up}

Patients were followed postoperatively at 6 weeks and 3 months by their usual endocrinologist. Subsequent follow-up was scheduled based upon hormone profile and clinical need. In some cases, particularly complex patients, our endocrine team remained intimately involved in the care of the patient either through telemedicine or alternating appointments with their local endocrinologist.

\section{Surgical Follow-up}

All patients were followed-up at 3 weeks, 3 months, and 12 months, and thereafter as required based on concerns of residual or recurrent disease. Repeat magnetic resonance imaging (MRI) was obtained at 3 months and 12 months and 
thereafter as directed by endocrine care or MDT recommendation.

\section{Population Characteristics}

Age, gender, tumor subtype (determined by hormone immunohistochemistry), tumor size, parasellar involvement (cavernous, sphenoid sinus, or suprasellar), preoperative medical therapy and pituitary hormone replacement, number of previous pituitary surgeries, and surgical approach (endoscopic or microscopic), as well as the names of the treating endocrinologist, neurosurgeon, and otolaryngologist, were collected.

\section{Perioperative Outcomes (Less Than 30 Days Postsurgery)}

Data collected included length of hospital stay, unplanned representation to hospital, endocrine complications (transient DI and SIADH), surgical complications (cerebrospinal fluid [CSF] leak, epistaxis, intracranial hemorrhage, meningitis, and mortality), and return to theater. Transient DI was defined as requiring synthetic antidiuretic hormone (desmopressin) within 1 week from surgery but not beyond the first week. SIADH was defined by hyponatremia within 2 weeks postoperatively (mild if serum sodium was 130 -$135 \mathrm{mmol} / \mathrm{L}$ at lowest, moderate if $120-130 \mathrm{mmol} / \mathrm{L}$ and severe if $<120 \mathrm{mmol} / \mathrm{L}$ ). CSF leak, epistaxis, and intracranial hemorrhage were recorded if requiring return to theater. Meningitis was defined as requiring antibiotic therapy after diagnosis by the surgical team.

\section{Postoperative Outcomes (More Than 30 Days Postsurgery)}

Data collected included presence and location of residual tumor as determined by 3-month postoperative MRI, new visual deficit as defined by an ophthalmologist, new endocrine deficiencies (permanent DI, hypocortisolaemia, hypothyroidism, gonadotropin [Gn], and growth hormone [GH] deficiency), reoperation within 1 year and adjuvant radiotherapy within 1 year. Unexpected intrasellar residual is defined as residual within the sella, on the 3-month postsurgery MRI, in cases where the preoperative plan was gross total resection. In cases of planned subtotal resection, such as cavernous sinus invasion, the residual component was not included in this definition. New endocrine deficiencies were defined by treatment specific criteria for the purposes of defining permanent DI (requiring desmopressin for at least 1 month postoperatively), hypocortisolaemia (requiring glucocorticoid therapy beyond 6 months of surgery), and hypothyroidism (requiring thyroid hormone replacement within the first year after surgery). New-onset Gn deficiency was defined within 1 year of surgery; in men with low testosterone, in noncycling premenopausal women with low oestradiol, or in postmenopausal women in whom low/normal follicle stimulating or luteinizing hormone developed. Finally, new-onset GH deficiency was defined if insulin-like growth factor 1 (IGF-1) was low within 1 year after surgery, in the presence of other pituitary deficiencies or as determined by dynamic testing.

\section{Statistical Analysis}

Statistical analysis was performed using SPSS v24 (SPSS Inc., Chicago). Nominal variables were compared using the Pearson Chi-square test unless subgroup sample sizes were sufficiently small requiring Fisher's exact test. Ordinal variables were compared by the independent samples MannWhitney $U$-test. Scalar variables were compared by the independent samples $t$-test if normally distributed and by the independent samples Mann-Whitney $U$-test if nonparametrically distributed. Binomial logistic regression was used to perform multivariate analysis when determining overall complication status. The impact on complication rate was reported as an odds ratio (OR) and a 95\% confidence interval (CI). Null hypotheses were rejected where $p<0.05$.

\section{Results}

\section{Population Characteristics}

The study cohort comprised 279 pituitary surgery cases (89 patients of pre-MDT and 190 patients of post-MDT), age $54 \pm 17$ years, $48 \%$ female. Pituitary tumors comprised $92 \%$ of the cohort. Clinically nonfunctioning adenoma was the most common tumor subtype (54\%), followed by acromegaly (18\%), prolactinoma (11\%), Cushing's disease (9\%), and thyroid stimulating hormone (TSH)-secreting adenoma (1\%). Rathke's cleft cysts were the most frequent nontumor pathology (4\%), with other histopathology including meningiomas, craniopharyngiomas, juvenile nasopharyngeal angiofibromas, metastatic melanoma, hypophysitis, and pituitary abscess.

On preoperative imaging $81 \%$ of tumors were between 10 and $40 \mathrm{~mm}$ in maximal diameter. More than half (56\%) caused optic chiasm compression, while cavernous or sphenoid sinus invasion was seen in 36 and 13\% of tumors, respectively. There were fewer macroadenomas (85.3 vs. $90.4 \%, p=0.26$ ), less cavernous sinus involvement (30 vs. $48 \%, p<0.01$ ) and optic chiasm compression (47 vs. $77 \%$, $p<0.01$ ) in the post-MDT group (-Table $\mathbf{1}$ ).

There was a higher proportion of clinically functioning tumors in the post-MDT group (42 vs. $28 \%, p=0.03$ ). Less than one-fifth of all pituitary tumors received preoperative medical therapy, specifically dopamine agonists (14\%) and somatostatin analogues (3\%), with no significant difference in use of preoperative medical therapy between pre- and post-MDT groups. One-third of patients were on hormone replacement therapy preoperatively in both pre- and postMDT groups, thyroid hormone and glucocorticoids most commonly (63 and 61\%, respectively).

The minority of cases were revision surgeries (22\%) and, in most cases, a total resection ( $80 \%$ ) was attempted as opposed to a partial resection. Curative resections were attempted more commonly post-MDT (87 vs. $66 \%, p<0.01$ ). Surgical technique was primarily endoscopic endonasal transsphenoidal surgery (83\%). Microscopic endonasal surgery was performed in $11 \%$, with subfrontal and sublabial approaches performed less frequently. A greater number of cases were performed endoscopically post-MDT (98 vs. $46 \%, p<0.001$ ), although the transsphenoidal approach was clearly the most common in both groups (99 vs. $92 \%, p<0.01$ ). 
Table 1 Comparison of baseline characteristics between pre- and post-MDT groups

\begin{tabular}{|c|c|c|c|}
\hline & Pre-MDT $(n=89)$ & Post-MDT $(n=190)$ & p-Value \\
\hline Age at surgery (mean, SD) & $54 \pm 16$ & $54 \pm 18$ & $0.75^{\mathrm{a}}$ \\
\hline Female (\%) & 38.2 & 52.6 & $0.03^{b}$ \\
\hline Diagnosis & & & $0.65^{b}$ \\
\hline Nonfunctioning adenoma (\%) & 62.5 & 50.0 & \multirow[t]{6}{*}{$N / A$} \\
\hline Acromegaly (\%) & 14.8 & 17.4 & \\
\hline Prolactinoma (\%) & 6.8 & 13.2 & \\
\hline Cushing's disease (\%) & 8.0 & 8.9 & \\
\hline Rathke’s cyst (\%) & 4.5 & 3.7 & \\
\hline Miscellaneous (\%) & 3.4 & 6.3 & \\
\hline Tumor size & & & $0.26^{\mathrm{b}}$ \\
\hline Micro <10 mm (\%) & 9.6 & 14.7 & \multirow[t]{2}{*}{$\mathrm{N} / \mathrm{A}$} \\
\hline Macro $10-40$ mm (\%) & 90.4 & 85.3 & \\
\hline \multicolumn{4}{|l|}{ Parasellar Involvement (\%) } \\
\hline Cavernous sinus & 48.1 & 30.1 & $<0.01^{\mathrm{b}}$ \\
\hline Sphenoid sinus & 18.4 & 10.3 & $0.08^{b}$ \\
\hline Chiasm compression & 77.0 & 47.4 & $<0.01^{\mathrm{b}}$ \\
\hline Hormone secreting (\%) & 28.2 & 42.2 & $0.03^{b}$ \\
\hline Preoperative medical therapy (\%) & 11.4 & 21.2 & $0.05^{b}$ \\
\hline Preoperative hormone replacement (\%) & 40.9 & 32.8 & $0.19^{b}$ \\
\hline Primary surgery (\%) & 77.3 & 78.4 & $0.83^{\mathrm{b}}$ \\
\hline Gross total resection (\%) & 65.9 & 87.1 & $<0.01^{\mathrm{b}}$ \\
\hline Surgical technique (\%) & & & $<0.01^{\mathrm{b}}$ \\
\hline Endoscopic endonasal & 46.4 & 98.4 & \multirow[t]{2}{*}{$\mathrm{N} / \mathrm{A}$} \\
\hline Other (microscopic transnasal, sublabial, subfrontal) & 53.6 & 1.6 & \\
\hline
\end{tabular}

Abbreviations: MDT, multidisciplinary team; N/A, not available; SD, standard deviation.

andependent Samples Student's t-test.

bPearson's Chi-square test.

${ }^{\mathrm{C}}$ Mann-Whitney U-test.

Prior to commencement of the MDT, pituitary surgery was performed by seven neurosurgeons and three otolaryngologists. Following the introduction of the MDT, $80 \%$ of pituitary surgery was performed by 2 neurosurgeons with $98 \%$ of cases involving one otolaryngologist. Baseline characteristics were otherwise similar between pre- and postMDT groups.

\section{Perioperative Patient Outcomes ( $<30$ Days)}

Length of perioperative hospital stay was shorter among post-MDT compared with pre-MDT cases (5 [range: 4-7] vs. 7 days [range: $6-11$ ], $p<0.001$; - Fig. 1). Unexpected representation to hospital occurred in $10 \%$ of cases $(9.1 \%$ post-MDT vs. $12.5 \%$ pre-MDT, $p>0.05$ ), requiring readmission in most of those (86\%; - Table 2). Representation rates were similar post- and pre-MDT, most commonly due to SIADH (67 and $40 \%$ ) followed by epistaxis (17 and 20\%). Hypocortisolaemia, hypothyroidism, hydrocephalus, and CSF leak caused one representation each. CSF leak occurred in $4 \%$ of cases, epi-

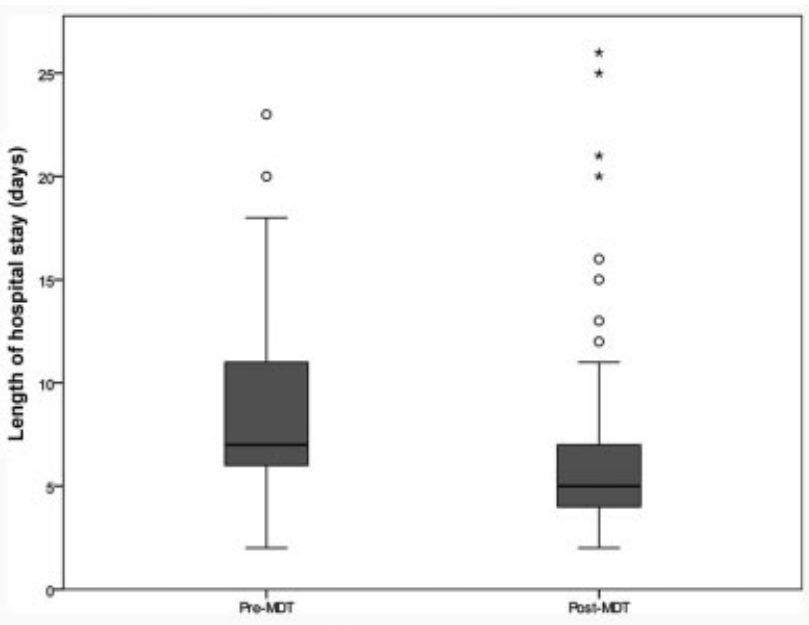

Fig. 1 Comparison of perioperative length of hospital stay in days before and after multidisciplinary team (MDT) introduction. 
Table 2 Comparison of perioperative outcomes (within 30 days) between pre- and post-MDT groups

\begin{tabular}{|c|c|c|c|}
\hline & Pre-MDT $(n=89)$ & Post-MDT $(n=190)$ & $p$-Value \\
\hline Length of hospital stay (d) & $7(5)$ & $5(3)$ & $<0.01^{\mathrm{a}}$ \\
\hline Unplanned representation (\%) & 12.5 & 9.1 & $0.39^{b}$ \\
\hline \multicolumn{4}{|l|}{ Endocrinological } \\
\hline Transient DI (\%) & 36.4 & 19.9 & $<0.01^{\mathrm{b}}$ \\
\hline SIADH (\%) & 38.6 & 17.7 & $<0.01^{d}$ \\
\hline Mild (\%) & 28.4 & 11.8 & \multirow[t]{3}{*}{$\mathrm{N} / \mathrm{A}$} \\
\hline Moderate (\%) & 6.8 & 4.3 & \\
\hline Severe (\%) & 3.4 & 1.6 & \\
\hline \multicolumn{4}{|l|}{ Surgical } \\
\hline CSF leak (\%) & 4.5 & 4.3 & $1.00^{c}$ \\
\hline Epistaxis (\%) & 1.1 & 1.1 & $1.00^{c}$ \\
\hline Intracranial hemorrhage (\%) & 1.1 & 1.6 & $1.00^{c}$ \\
\hline Meningitis (\%) & 2.3 & 2.2 & $1.00^{c}$ \\
\hline Mortality (\%) & 0 & 0 & $\mathrm{~N} / \mathrm{A}$ \\
\hline New visual deficit (\%) & 1.1 & 1.6 & $1.00^{c}$ \\
\hline Return to theater (\%) & 7.0 & 10.2 & $0.40^{\mathrm{b}}$ \\
\hline
\end{tabular}

Abbreviations: CSF, cerebrospinal fluid; DI, diabetes insipidus; MDT, multidisciplinary team; N/A, not available; SIADH, syndrome of inappropriate antidiuretic hormone.

andependent Samples Mann-Whitney U-test.

'Pearson's Chi-square test.

'Fisher's exact test.

'Kendall's tau B.

staxis $1 \%$, intracranial hemorrhage $2 \%$, and meningitis $2 \%$, at similar rates pre- and post-MDT. New visual deficits were present in $2 \%$ of cases, due to cranial nerve III or VI palsy. Unplanned return to theater within 30 days of surgery was required in $9 \%$ of all cases, due to definite residual tumor on immediate postoperative imaging or previously stated complications. There was no perioperative mortality.

Transient DI occurred in $25 \%$ of cases and was less common post-MDT ( 20 vs. $36 \%, p<0.01$ ). SIADH was also diagnosed in $25 \%$ of cases, most instances were mild (70\%), with moderate and severe hyponatraemia in 21 and 9\%, respectively. SIADH was also less common post-MDT (18 vs. $39 \%, p<0.01)$.

\section{Postoperative Outcomes (More than 30 Days Postsurgery)}

New anterior pituitary hormone deficits were seen in $16 \%$ (most commonly hypocortisolaemia $>$ hypothyroidism $>$ Gn

Table 3 Comparison of long term postoperative outcomes (>30 days) between pre- and post-MDT groups

\begin{tabular}{|l|l|l|l|}
\hline & Pre-MDT $(\boldsymbol{n}=89)$ & Post-MDT $(\boldsymbol{n}=190)$ & $p$-Value \\
\hline Intrasellar residual (\%) & 34.8 & \multicolumn{2}{l|}{} \\
\hline Endocrinological & \multicolumn{2}{|l|}{$<0.001^{\mathrm{a}}$} \\
\hline Permanent DI (\%) & 8.0 & 3.2 & $0.13^{\mathrm{b}}$ \\
\hline Hypocortisolaemia (\%) & 14.8 & 8.6 & $0.14^{\mathrm{b}}$ \\
\hline Hypothyroidism (\%) & 14.8 & 4.8 & $<0.01^{\mathrm{b}}$ \\
\hline Gn deficiency (\%) & 6.8 & 4.3 & $0.39^{\mathrm{b}}$ \\
\hline GH deficiency (\%) & 2.3 & 0 & $0.10^{\mathrm{b}}$ \\
\hline Reoperation within 1 year (\%) & 6.8 & 2.6 & $0.11^{\mathrm{b}}$ \\
\hline Radiotherapy (\%) & 11.4 & 6.4 & $0.16^{\mathrm{b}}$ \\
\hline cFree of complication (\%) & 31.0 & 58.6 & $<0.001^{\mathrm{b}}$ \\
\hline
\end{tabular}

Abbreviations: DI, diabetes insipidus; GH, growth hormone; Gn, gonadotropin; MDT, multidisciplinary team.

a'Pearson's Chi-squared test.

${ }^{b}$ Fischer's exact test.

${ }^{\mathrm{C}}$ Free of all peri- and postoperative complications. 
Table 4 factors influencing any peri- or postoperative complication

\begin{tabular}{|l|l|l|}
\hline \multicolumn{3}{|l|}{ Risk of complication during pituitary care } \\
\hline $\begin{array}{l}\text { Treatment and disease } \\
\text { factors }\end{array}$ & OR $(95 \% \mathrm{CI})$ & $p$-Value \\
\hline Pre-MDT & $2.13(1.05-4.32)$ & $0.04^{\mathrm{a}}$ \\
\hline Microadenoma & $1.19(0.52-2.72)$ & $0.68^{\mathrm{a}}$ \\
\hline $\begin{array}{l}\text { Endoscopic only } \\
\text { surgical access }\end{array}$ & $0.51(0.21-1.27)$ & $0.15^{\mathrm{a}}$ \\
\hline Nonfunctioning adenoma & $1.24(0.69-2.24)$ & $0.47^{\mathrm{a}}$ \\
\hline
\end{tabular}

Abbreviations: $\mathrm{Cl}$, confidence interval; MDT, multidisciplinary team.

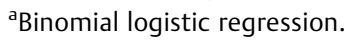

deficiency $>$ GH deficiency $>$ combinations). Permanent DI was seen in 5\%. Reoperation within 1 year was required in $4 \%$ of cases and adjuvant radiotherapy in $8 \%$.

Among the long-term outcomes ( $>30$ days postsurgery), there were fewer intrasellar residuals in the post-MDT group (8.4 vs. 35.0\%, $p<0.001$; - Table 3). New hypothyroidism was also less common in the post-MDT group (5.0 vs. $15 \%$, $p<0.01)$. Other outcomes were similar between the groups.

More cases of post-MDT were free of any peri- or postoperative endocrine or surgical complication (59 vs. $31 \%$, $p<0.001)$. This difference was retained on multivariate regression analysis including MDT group, tumor size, hormone secreting status, and surgical technique (odds ratio $[\mathrm{OR}]=2.13$ [1.05-4.32], $p=0.04$; - Table 4 and -Fig. 2).

\section{Discussion}

Since the introduction of a pituitary MDT clinic, in our institution, there has been benefit in reduction of inpatient hospital days, less transient DI, less SIADH, less hypothyroidism, less unexpected residual tumor, and overall less complication. This study was implemented and the original motivation to create a pituitary MDT was out of convenience for the involved specialists and patients and less about improving outcomes. However, after several years, it was clear that patient outcomes were improving beyond changes in technology or medication options alone.

However, there were several patient and treatment factors that have changed in the post-MDT era. Notably, the shift to endoscopic only surgical approach (46.4 vs. $96.4 \%$, $p<0.01$ ), more functional tumors undergoing surgical intervention ( 28.2 vs. $42.2 \%, p=0.03$ ), higher case load ( 5 years either side: $n=190$ vs. $n=89$ ), and fewer participating surgeons with two surgeons performing the majority of cases post-MDT (78.2 vs. $56.2 \%, p<0.01$ ). There was also an increased rate of gross-total resection (GTR) in the postMDT era. This increase may be a reflection of the increased number of functional tumors that were treated post-MDT, as the goal for these patients would include emphasis on GTR of the tumor. As a direct result of the MDT, tumors were being discussed earlier and regularly during their management and were potentially referred for surgical intervention sooner in the treatment paradigm. The MDT institution also culled out the "occasional" pituitary surgeon as they did not attend meetings, were not comfortable meeting the standard or request of the MDT, and subsequently referral patterns changed. This in turn led higher case-loads for the participating surgeons and potentially skill improvement. This occurred in both neurosurgical and otolaryngology surgeons in our study. High volume surgical centers with higher individual surgeon caseloads have been associated with lower postoperative complication rates, ${ }^{28}$ but the concept of achieving a better endocrinological outcome or GTR is as much a product of the MDT involvement and expectations that are set for a patient care pathway, in our opinion.

Less transient DI, SIADH, and permanent hypothyroidism postpituitary surgery was found post-MDT. Rates of CSF leak, epistaxis, severe intraoperative hemorrhage, meningitis, new visual deficits, and intraoperative mortality were in line with rates reported in the literature. ${ }^{22,29-33}$ In the postMDT era, more patients were free of any complication and length of hospital stay was shorter.

Following the introduction of an MDT, a lower rate of intrasellar residual tumor was detected on the 3-month postoperative MRI, consistent with the increased number of surgeries post-MDT with curative intent. This is likely a
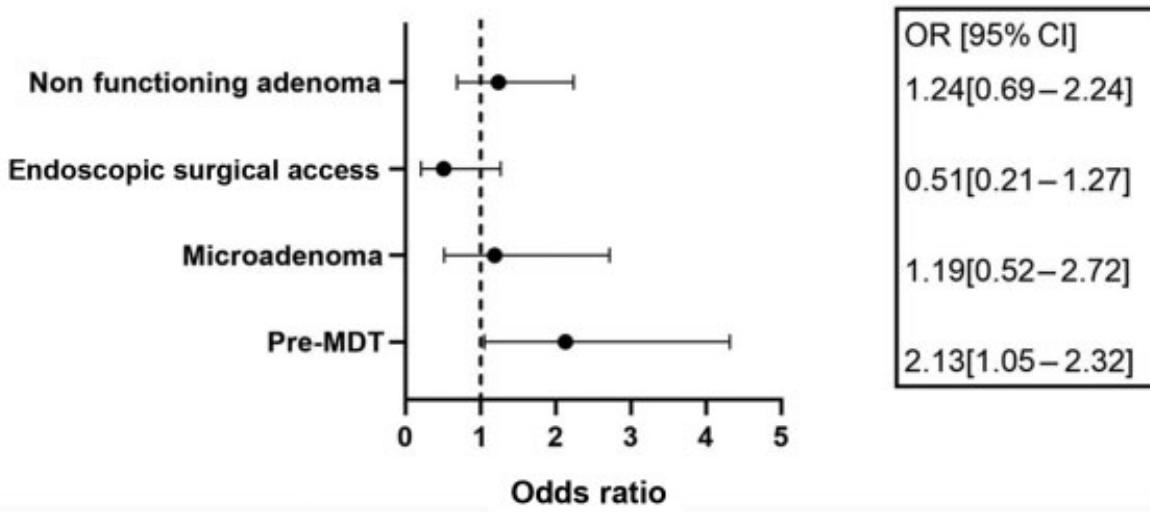

Fig. 2 Factors influencing any perioperative or postoperative complications 
combination of the skills of more dedicated pituitary surgeons needing to meet the MDT's expectations. Similar care models, in the United Kingdom, in which one to two pituitary surgeons per center, undertaking 30 to 40 operations per year are established. ${ }^{34,35}$ All of these findings suggest a correlation between the introduction of an MDT and is not explained simply by increased endoscopic surgical technique, as this is not a significant factor in regression analysis, (pre-MDT, OR = 2.13[1.05-4.32]; $p=0.04$ ).

Multidisciplinary collaboration is likely to allow for better awareness and adoption of technical and conceptual advances, ${ }^{14}$ and integration of these into appropriate treatment algorithms. This has already been demonstrated for endoscopic techniques and newer medical and radiographic therapies. ${ }^{23,36}$ Centers where pituitary surgery is currently performed at a high volume may benefit from the introduction of an MDT or consider referral to MDT driven centers as suggested in the Pituitary Society Centers of Excellence guidelines. ${ }^{9}$

\section{Conclusion}

Outcomes of pituitary surgery were demonstrated improvement after introduction of an MDT at a single center. This included increased curative resection rates, lower complication rates, and shorter hospital stays.

\section{Conflict of Interest}

R.J.H. is a consultant with Medtronic, Olympus and NeilMed pharmaceuticals. He has also been on the speakers' bureau for Glaxo-Smith-Kline, Seqirus and AstraZeneca. A.M. has received speaker fees from and participated as an advisory board member for Novartis, Ipsen and Pfizer. M.W. is a consultant for Nuvasive Pty Ltd. B.J. has consulted for Integra LifeSciences.

\section{Acknowledgments}

The authors acknowledge student contributions by Ian Matchett, Adam Wilkinson, and Maria Joseph to the St. Vincent's pituitary research database.

\section{References}

1 Fleissig A, Jenkins V, Catt S, Fallowfield L. Multidisciplinary teams in cancer care: are they effective in the UK? Lancet Oncol 2006;7 (11):935-943

2 Guidelines for Developing HealthOne NSW Services. Available at: https://www.health.nsw.gov.-

au/healthone/Documents/honswguidedevelop_11.pdf. Accessed September 27, 2019.

3 National Institute for Health and Clinical Excellence. Improving Outcomes for People with Brain and Other CNS Tumours: The Manual. London: National Institute for Health and Clinical Excellence; 2006

4 Linck P, Priedane E, Hughes DA, Edwards RT. Analysis of the Potential Economic Impact of the Guidance: 'Improving Outcomes for People with Brain and Other CNS Tumours. London, UK: National Institute for Health and Clinical Excellence; 2006

5 Taylor C, Munro AJ, Glynne-Jones R, et al. Multidisciplinary team working in cancer: what is the evidence? BMJ 2010;340: c951
6 Rainsbury JW, Ginn E, De R, Ahmed SK, Irving RM. The skull base multidisciplinary team approach: our experience over the first year in three hundred and seventeen patients. Clin Otolaryngol 2012;37(06):470-474

7 Casanueva FF, Barkan AL, Buchfelder M, et al; Pituitary Society, Expert Group on Pituitary Tumors. Criteria for the definition of pituitary tumor centers of excellence (PTCOE): a pituitary society statement. Pituitary 2017;20(05):489-498

8 Inder WJ, Alford FP. Pituitary masses: the importance of a multidisciplinary approach. Med J Aust 2007;187(09):522-523

9 Charalampaki P, Ayyad A, Kockro RA, Perneczky A. Surgical complications after endoscopic transsphenoidal pituitary surgery. J Clin Neurosci 2009;16(06):786-789

10 Hendricks BL, Shikary TA, Zimmer LA. Causes for 30-day readmission following transsphenoidal surgery. Otolaryngol Head Neck Surg 2016;154(02):359-365

11 Johnston PC, Kennedy L, Hamrahian AH, et al. Surgical outcomes in patients with Cushing's disease: the Cleveland clinic experience. Pituitary 2017;20(04):430-440

12 Joshi SM, Cudlip S. Transsphenoidal surgery. Pituitary 2008;11 (04):353-360

13 Yuan W. Managing the patient with transsphenoidal pituitary tumor resection. J Neurosci Nurs 2013;45(02):101-107, quiz E1E2

14 Laws ER Jr, Iuliano SL, Cote DJ, Woodmansee W, Hsu L, Cho CH. A benchmark for preservation of normal pituitary function after endoscopic transsphenoidal surgery for pituitary macroadenomas. World Neurosurg 2016;91:371-375

15 Lund-Johansen M. Neurosurgical endocrinology, endocrinological neurosurgery and interdisciplinary work. World Neurosurg 2015;83(05):765-766

16 McLaughlin N, Kassam AB, Prevedello DM, Kelly DF. Management of Cushing's disease after failed surgery-a review. Can J Neurol Sci 2011;38(01):12-21

17 Melmed S. Pituitary medicine from discovery to patient-focused outcomes. J Clin Endocrinol Metab 2016;101(03):769-777

18 Melmed S, Casanueva F, Cavagnini F, et al. Consensus statement: medical management of acromegaly. Eur J Endocrinol 2005;153 (06):737-740

19 Melmed S, Colao A, Barkan A, et al; Acromegaly Consensus Group. Guidelines for acromegaly management: an update. J Clin Endocrinol Metab 2009;94(05):1509-1517

20 Prevedello DM, Pouratian N, Sherman J, et al. Management of Cushing's disease: outcome in patients with microadenoma detected on pituitary magnetic resonance imaging. J Neurosurg 2008;109(04):751-759

21 Rajasekaran S, Vanderpump M, Baldeweg S, et al. UK guidelines for the management of pituitary apoplexy. Clin Endocrinol (Oxf) 2011;74(01):9-20

22 Singer PA, Sevilla LJ. Postoperative endocrine management of pituitary tumors. Neurosurg Clin N Am 2003;14(01):123-138

23 Swearingen B. Update on pituitary surgery. J Clin Endocrinol Metab 2012;97(04):1073-1081

24 Vargas G, Gonzalez B, Ramirez C, et al. Clinical characteristics and treatment outcome of 485 patients with nonfunctioning pituitary macroadenomas. Int J Endocrinol 2015;2015:756069

25 McLaughlin N, Martin NA, Upadhyaya P, et al. Assessing the cost of contemporary pituitary care. Neurosurg Focus 2014;37(05):E7

26 Murad MH, Fernández-Balsells MM, Barwise A, et al. Outcomes of surgical treatment for nonfunctioning pituitary adenomas: a systematic review and meta-analysis. Clin Endocrinol (Oxf) 2010;73(06):777-791

27 Villwock JA, Villwock M, Deshaies E, Goyal P. Significant increases of pituitary tumors and resections from 1993 to 2011. Int Forum Allergy Rhinol 2014;4(09):767-770

28 Little AS, Chapple K. Improved resource utilization is associated with high volume surgical centers, low complication rates, and shorter length of stay in patients undergoing 
transsphenoidal surgery for cushing's disease. Neurosurgery 2012;71(02):E548

29 Gondim JA, Almeida JP, de Albuquerque LA, Gomes E, Schops M, Mota JI. Endoscopic endonasal transsphenoidal surgery in elderly patients with pituitary adenomas. J Neurosurg 2015;123(01): 31-38

30 Patil CG, Lad SP, Harsh GR, Laws ER Jr, Boakye M. National trends, complications, and outcomes following transsphenoidal surgery for Cushing's disease from 1993 to 2002. Neurosurg Focus 2007; 23(03):E7

31 Raappana A, Koivukangas J, Ebeling T, Pirilä T. Incidence of pituitary adenomas in Northern Finland in 1992-2007. J Clin Endocrinol Metab 2010;95(09):4268-4275

32 Chowdhury T, Prabhakar H, Bithal PK, Schaller B, Dash HH. Immediate postoperative complications in transsphenoidal pitu- itary surgery: a prospective study. Saudi J Anaesth 2014;8(03): 335-341

33 Bohl MA, Ahmad S, Jahnke H, et al. Delayed hyponatremia is the most common cause of 30-day unplanned readmission after transsphenoidal surgery for pituitary tumors. Neurosurgery 2016;78(01):84-90

34 Bates PR, Carson MN, Trainer PJ, Wass JA; UK National Acromegaly Register Study Group (UKAR-2). Wide variation in surgical outcomes for acromegaly in the UK. Clin Endocrinol (Oxf) 2008;68 (01):136-142

35 Powell M, Grossman A. Quality indicators in pituitary surgery: a need for reliable and valid assessments. What should be measured? Clin Endocrinol (Oxf) 2016;84(04):485-488

36 Kreutzer J, Fahlbusch R. Diagnosis and treatment of pituitary tumors. Curr Opin Neurol 2004;17(06):693-703 